\title{
Iron metabolism disturbances in the MCF-7 human breast cancer cells with acquired resistance to doxorubicin and cisplatin
}

\author{
VASYL F. CHEKHUN ${ }^{1}$, NATALIA Yu. LUKYANOVA ${ }^{1}$, ANATOLIY P. BURLAKA ${ }^{1}$, NATALIA A. BEZDENEZHNYKH ${ }^{1}$, \\ SVITLANA I. SHPYLEVA ${ }^{2}$, VOLODYMYR P. TRYNDYAK ${ }^{1,2}$, FREDERICK A. BELAND ${ }^{2}$ and IGOR P. POGRIBNY ${ }^{2}$ \\ ${ }^{1}$ Department of Mechanisms of Anticancer Therapy, R.E. Kavetsky Institute of Experimental Pathology, Oncology and \\ Radiobiology, Kiev, Ukraine; ${ }^{2}$ Division of Biochemical Toxicology, National Center for Toxicological Research, \\ US Food and Drug Administration, Jefferson, AR 72079, USA
}

Received May 20, 2013; Accepted June 24, 2013

DOI: $10.3892 /$ ijo.2013.2063

\begin{abstract}
The development of resistance of cancer cells to therapeutic agents is the major obstacle in the succesful treatment of breast cancer and the main cause of breast cancer recurrence. The results of several studies have demonstrated an important role of altered cellular iron metabolism in the progression of breast cancer and suggested that iron metabolism may be involved in the acquisition of a cancer cell drug-resistant phenotype. In the present study, we show that human MCF-7 breast cancer cells with an acquired resistance to the chemotherapeutic drugs doxorubicin (MCF-7/DOX) and cisplatin (MCF-7/CDDP) exhibited substantial alterations in the intracellular iron content and levels of iron-regulatory proteins involved in the cellular uptake, storage and export of iron, especially in profoundly increased levels of ferritin light chain (FTL) protein. The increased levels of FTL in breast cancer indicate that FTL may be used as a diagnostic and prognostic marker for breast cancer. Additionally, we demonstrate that targeted downregulation of FTL protein by the microRNA miR-133a increases sensitivity of MCF-7/DOX and MCF-7/ CDDP cells to doxorubicin and cisplatin. These results suggest that correction of iron metabolism abnormalities may substantially improve the efficiency of breast cancer treatment.
\end{abstract}

\section{Introduction}

Breast cancer is the most prevalent malignancy in women (1). Despite a statistically significant decline in breast cancer incidence and death rate in recent years, breast cancer is still the leading cause of cancer death among women worldwide $(1,2)$ and second cause of cancer death among women in the US (1). Furthermore, $\sim 30 \%$ of patients with early-stage of breast cancer have recurrent disease (3). One of the major obstacles in the succesful treatment of breast cancer and the main cause

Correspondence to: Dr Igor P. Pogribny, Division of Biochemical Toxicology, NCTR, 3900 NCTR Rd, Jefferson, AR 72079, USA E-mail: igor.pogribny@fda.hhs.gov

Key words: breast cancer, iron metabolism, drug resistance of breast cancer recurrence is the resistance of cancer cells to therapeutic agents (3).

Several reports have provided compelling evidence for the connection between profoundly deregulated cellular iron metabolism and breast cancer progression (4-7). More importantly, the results of two recent comprehensive studies conducted by Pinnix et al (4) and Miller et al (6) demonstrated that iron regulatory gene signatures may predict breast cancer outcome. This suggests that characterizing the disturbances in iron metabolism in cancer cells, in addition to providing diagnostic and prognostic value, may be also potential therapeutic strategies for breast cancer treatment (8-10). Additionally, a report by Whitnall et al (11) has demonstrated that alterations in cellular iron metabolism may contribute to the acquisition of a cancer cell drug-resistant phenotype. The latter was evident from the data showing that treatment of the human etoposide-resistant MCF-7 breast cancer cells with iron-chelating agents reversed the resistance of cancer cells to chemotherapeutic agents (11); however, there is a lack of conclusive information regarding the dysregulation of iron metabolism in drug-resistant breast cancer cells.

The present study was undertaken to investigate the status and role of iron metabolism in drug-resistant breast cancer cells. We demonstrate that human MCF-7 breast cancer cells with an acquired resistance to the chemotherapeutic agents doxorubicin and cisplatin exhibit extensive alterations in the cellular iron homeostasis as characterized by marked changes in the level of intracellular 'free iron', a high-spin form of iron [Fe(III)] detectable by electron paramagnetic resonance (EPR) and proteins responsible for the cellular uptake, storage and export of iron, especially by profound upregulation of ferritin light chain (FTL) protein. Furthermore, the results demonstrate that targeted downregulation of overexpressed FTL protein by microRNA miR-133a increases the sensitivity of drug-resistant cells to doxorubicin and cisplatin. This suggests that correcting of intracellular iron metabolism may be a potential approach to overcome resistance of breast cancer cells to chemotherapeutic agents.

\section{Materials and methods}

Cell lines and cell culture. The human breast adenocarcinoma MCF-7 cell line and its variants resistant to doxorubicin 
hydrochloride (MCF-7/DOX; resistance index, 5.6) or cisdiammineplatinum(II) dichloride (MCF-7/CDDP; resistant index, 6.0) were cultured in complete Dulbecco's modified Eagle's medium (DMEM; Sigma-Aldrich, St. Louis, MO, USA) containing $10 \%$ embryonal calf serum (Sigma-Aldrich) and $40 \mu \mathrm{g} / \mathrm{ml}$ gentamycin at $37^{\circ} \mathrm{C}$ in $5 \% \mathrm{CO}_{2}$ atmosphere. The drug-resistant variants of MCF-7 cell lines were established as described in Chekhun et al (12) and the resistant index was determined as outlined in Kars et al (13). Cells were seeded at a density of $0.5 \times 10^{6}$ viable cells per $100-\mathrm{mm}$ plate and the medium was changed every other day for 4 days.

Low-temperature $\mathrm{Fe}(I I I) E P R$. After $24 \mathrm{~h}$ of culturing in complete DMEM, the cells were scrapped onto ice, washed in phosphate-buffered saline (PBS), centrifuged at $1000 \mathrm{~g}$ for $10 \mathrm{~min}$ at $4^{\circ} \mathrm{C}$ and the pellet was re-suspended in PBS. The suspension containing $2 \times 10^{6}$ cells was transferred into EPR tubes and immediately frozen in liquid nitrogen. The level of free iron was determined by a low-temperature EPR method (14). Briefly, samples were maintained at $-196^{\circ} \mathrm{C}$ during recording of the spectra using a finger Dewar filled with liquid nitrogen. The following parameters were used for the lowtemperature EPR: sweep width $1525 \mathrm{G}$; frequency $9.15 \mathrm{GHz}$; microwave power $40 \mathrm{~mW}$; modulation amplitude $10.0 \mathrm{G}$; and modulation frequency $100 \mathrm{kHz}$. The g-value was calculated using the standard formula $g=h v / \beta H$, where $h$ is Planck's constant, $\mathrm{v}$ is the frequency, $\beta$ is the Bohr magneton and $\mathrm{H}$ is the external magnetic field at resonance.

Immunocytochemistry. The levels of transferrin receptor 1 (TFR1), ferritin light chain (FTL), ferritin heavy chain (FTH1) and ferroportin (FPN) were determined by immunocytochemistry. Cells were cultured on glass coverslips for $24 \mathrm{~h}$ and fixed in ice-cold methanol:acetone $(1: 1)$ at $-20^{\circ} \mathrm{C}$ for $10 \mathrm{~min}$. The fixed cells were then rinsed in PBS and after blocking of non-specific staining with a $1 \%$ BSA solution for $20 \mathrm{~min}$, the cells were incubated with primary rabbit anti-human antibodies against TFR1 (1:100; BS1620; Bioworld Technology, Minneapolis, MN, USA), FTL (1:500; ab69090; Abcam, Cambridge, MA, USA), FTH1 (1:150; GTX62020; GeneTex, Irvine, CA, USA) and FPN (1:50; ab78066; Abcam) at room temperature for $60 \mathrm{~min}$ followed by incubation with an UltraVision LP Detection System (Thermo Fisher Scientific, Waltham, MA, USA) for 15 min. Staining was developed with 3,3'-diaminobenzidine Quanto (Thermo Fisher Scientific). The cells were counterstained with hematoxylin. The staining intensity was evaluated by the $\mathrm{H}$-score method as described in McClelland et al (15). Briefly, the percentage of slightly (a), moderately (b) and strongly stained cells (c) was determined and then used in the formula $S=(1 \times a)+(2 \times b)+(3 \times c)$ to calculate an ' $\mathrm{H}$-score'. The $\mathrm{S}$ values ranged from 0 (no expression) to 300 (strong expression in $100 \%$ cells).

Drug sensitivity assay. To determine drug sensitivity, MCF-7, MCF-7/DOX and MCF-7/CDDP cells were plated at a density of 10,000 cells per well in 96-well plates. Cells were cultured for $24 \mathrm{~h}$ and then treated with doxorubicin, cisplatin, or bleomycin sulfate (Sigma-Aldrich). After 48- and 72-h incubation, cell survival was analyzed with sulforhodamine B (16) and MTT (3-(4,5-dimethylthiazol-2-yl)-2,5-diphenyltetrazolium

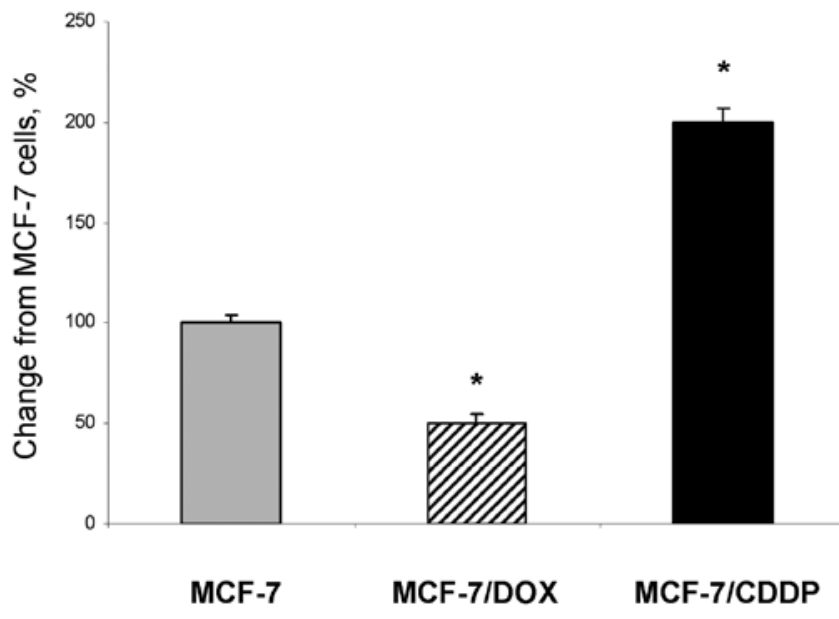

Figure 1. Levels of Fe(III) in MCF-7 and drug-resistant MCF-7/DOX and MCF-7/CDDP breast cancer cells. The level of intracellular unincorporated $\mathrm{Fe}$ (III) was determined by low-temperature EPR spectroscopy as detailed in Materials and methods. The data are presented as percent change in the $\mathrm{Fe}$ (III) level in MCF-7/DOX and MCF-7/CDDP cells relative to that in MCF-7 cells, which were assigned a value of $100 \%$. Asterisks (*), significantly different from MCF-7 cells $(n=5)$.

bromide) assays (17). The $\mathrm{IC}_{50}$ (inhibitory concentration to produce $50 \%$ cell death) values were determined from using the resulting dose-response curves. The experiments were repeated twice and each cell line was tested in triplicate.

Transfection of MCF-7/DOX, MCF-7/CDDP and MDA-MB231 cells with pre-miR-133a and siRNA-FTL. MCF-7/DOX, MCF-7/CDDP and MDA-MB-231 cells were seeded in $100-\mathrm{mm}$ dishes at a density of $1 \times 10^{6}$ cells per dish and transfected with $20 \mathrm{nM}$ of pre-miR-133a (Life Technologies, Grand Island, NY, USA) and $10 \mathrm{nM}$ of Silencer ${ }^{\circledR}$ Select siRNA-FTL (Life Technologies), in three independent replicates, using Lipofectamine $^{\mathrm{TM}} 2000$ (Life Technologies) transfection reagent according to the manufacturer's instructions. MCF-7/ DOX, MCF-7/CDDP and MDA-MB-231 cells transfected with scrambled RNA oligonucleotide served as controls. At $48 \mathrm{~h}$ post-transfection, adherent cells were harvested by mild trypsinization and the viability of cells was monitored by a MTT test. The cells were then re-seeded and the transfection repeated. Forty-eight hours after the second transfection, adherent cells were harvested by mild trypsinization, washed in PBS and frozen at $-80^{\circ} \mathrm{C}$ for subsequent analyses. The experiments were repeated twice.

Western blot analysis of protein expression. The level of FTL protein in the breast cancer cells was determined by western immunoblot analysis with primary antibodies against ferritin light chain (FTL; 1:200; Santa Cruz Biotechnology, Santa Cruz, CA, USA) as described in Shpyleva et al (18).

Statistical analyses. Statistical analysis was done using Statistica 7.0 software (StatSoft Inc., Tulsa, OK, USA). Results are presented as mean $\pm \mathrm{SD}$. Data were analyzed by one-way analysis of variance (ANOVA), with pair-wise comparisons being made by the Student-Newman-Keuls method. P-values $<0.05$ were considered statistically significant. 


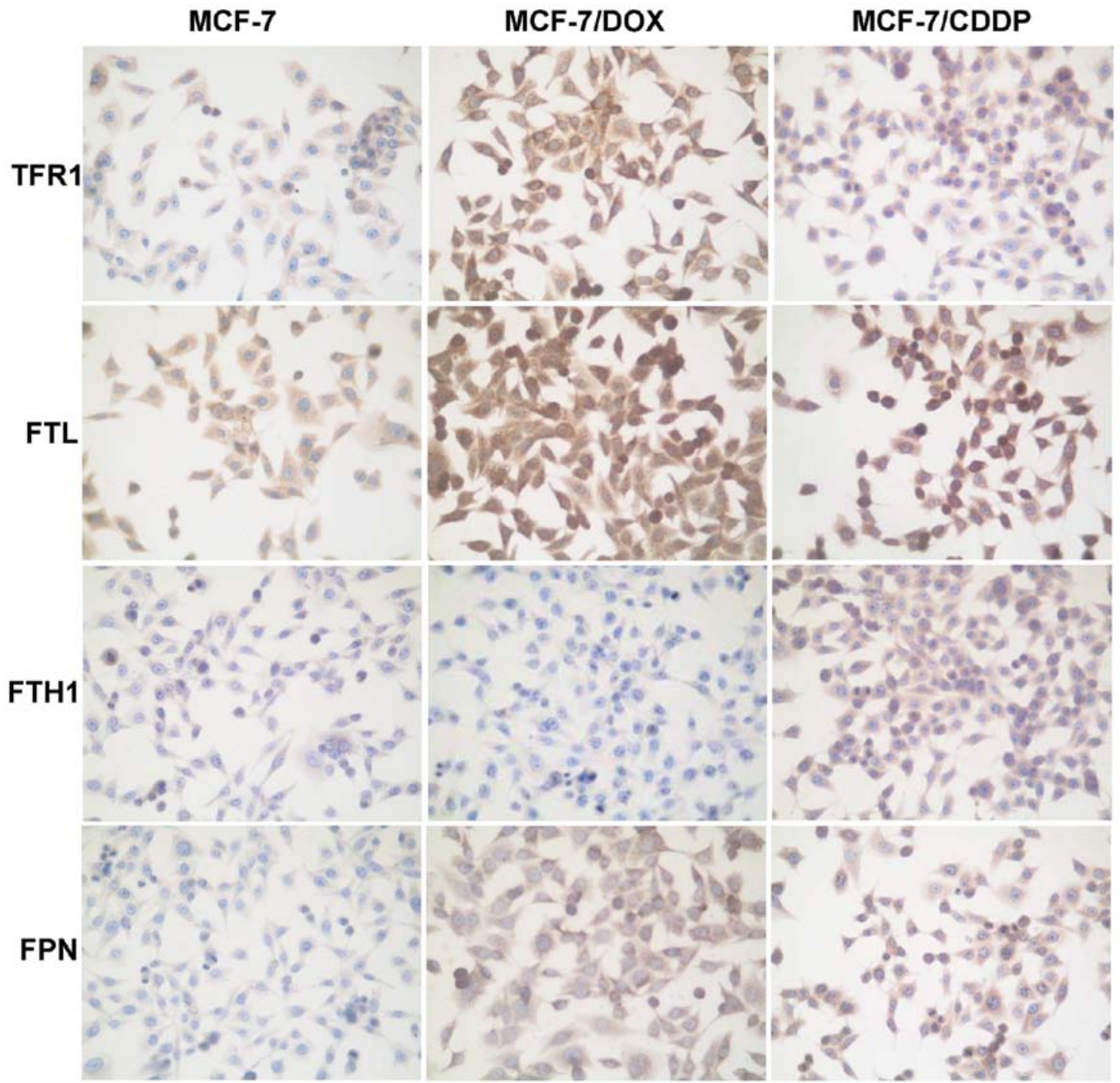

Figure 2. Staining for TFR1, FTL,FTH1 and FPN proteins in MCF-7 and drug-resistant MCF-7/DOX and MCF-7/CDDP breast cancer cells. Magnification, x40. Representative images are shown.

\section{Results}

Changes in intracellular Fe(IIl) in the MCF-7/DOX and $M C F-7 / C D D P$ resistant cancer cells. Fig. 1 shows that drug-resistant MCF-7/DOX and MCF-7/CDDP cells are characterized by a substantial variation in the 'free' intracellular Fe(III) content as compared to parental MCF-7 cells. Specifically, the level of intracellular Fe(IIl) in the MCF-7/ CDDP cells was 2.0 times greater than in MCF-7 cells. In contrast, the intracellular level of Fe(III) in MCF-7/DOX cells was 2.0 times lower than in parental MCF-7 cells.

Alterations of iron-regulatory proteins in the MCF-7/DOX and MCF-7/CDDP resistant cancer cells. Table I shows changes in the levels of proteins involved in the cellular uptake (TFR1), storage (FTL and FTH1) and export (FPN) of iron in MCF-7 cells and the MCF-7/DOX and MCF-7/CDDP drug-resistant variants. In the MCF-7/DOX and MCF-7/CDDP drug-resistant cells the levels of TFR1, FTL and FPN proteins were 3.0, 1.5 and 2.5 times greater than their values in the parental MCF-7 cells (Table I and Fig. 2).
Table I. The levels of TFR1, FTL, FTH1 and FPN proteins in parental MCF-7 breast cancer cells and its drug-resistant variants $\mathrm{MCF}-7 / \mathrm{DOX}$ and $\mathrm{MCF}-7 / \mathrm{CDDP}$.

\begin{tabular}{lrrr}
\hline & \multicolumn{3}{c}{ The level of protein expression, H-score } \\
\cline { 2 - 4 } Protein & \multicolumn{1}{c}{ MCF-7 } & MCF-7/DOX & MCF-7/CDDP \\
\hline TFR1 & $72 \pm 1.8$ & $205 \pm 5.4^{\mathrm{a}}$ & $215 \pm 5.5^{\mathrm{a}}$ \\
FTL & $195 \pm 4.5$ & $298 \pm 2.1^{\mathrm{a}}$ & $296 \pm 5.8^{\mathrm{a}}$ \\
FTH1 & $125 \pm 2.7$ & $79 \pm 0.8^{\mathrm{a}}$ & $220 \pm 6.1^{\mathrm{a}}$ \\
FPN & $83 \pm 1.1$ & $200 \pm 3.4^{\mathrm{a}}$ & $203 \pm 5.2^{\mathrm{a}}$ \\
\hline
\end{tabular}

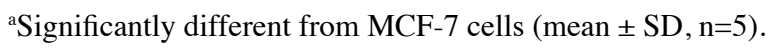

In contrast to the cell-type-independent changes in TFR1, FTL and FPN proteins in MCF-7/DOX and MCF-7/CDDP cells, alterations in the levels of FTH1 protein were cell-specific. This was evidenced by a $37 \%$ downregulation of FTH1 in the MCF-7/DOX cells as compared to the parental MCF-7 cells, 


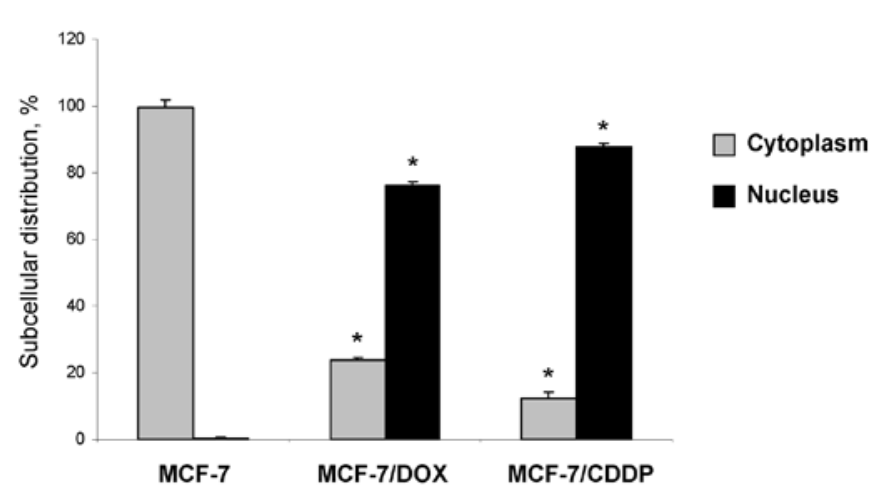

Figure 3. Distribution of FTL protein in MCF-7 and drug-resistant MCF-7/ DOX and MCF-7/CDDP breast cancer cells as detected by immunohistochemical staining and western immunoblotting. The data are presented as percent change in the levels of cytosolic and nuclear FTL protein in MCF-7/ DOX and MCF-7/CDDP cells relative to those in MCF-7 cells, which were assigned a value of $100 \%$. Asterisks (*), significantly different from MCF-7 cells $(n=5)$.

while in the MCF-7/CDDP, FTH1 was substantially (by 176\%) upregulated.

Intracellular localization of FTL protein in the MCF-7/DOX and $M C F-7 / C D D P$ resistant cancer cells. In our previous study we demonstrated that MDA-MB-231 breast cancer cells, which exhibit an advanced and intrinsic drug-resistant phenotype, were characterized by an upregulation of FTH1 and, especially, FTL proteins (18). Additionally, MDA-MB-231 breast cancer cells were characterized by increased levels of these proteins in their nuclei (18). This observation prompted us to investigate whether or not the upregulation of FTL in the MCF-7/DOX and MCF-7/CDDP resistant cancer cells is also accompanied by an altered subcellular distribution. It is well established that both FTH1 and FTL are primarily cytosolic proteins (19). Indeed, in parental MCF-7 cells, FTL is located only in the cytoplasm (Figs. 2 and 3). In contrast, in the MCF-7/DOX and MCF-7/CDDP resistant cancer cells, FTL was located primarily in the nucleus.

Sensitivity of the MCF-7/DOX and MCF-7/CDDP cells to chemotherapeutic agents. Having found significant changes in the levels of iron-regulatory proteins and intracellular Fe(III) content in the MCF-7/DOX and MCF-7/CDDP cells compare to MCF-7 cells, we investigated whether these changes were accompanied by a different resistance to other chemotherapeutic drugs, especially those in which the mechanism of action is linked to iron metabolism. Table II shows that the MCF-7/DOX cells, which are characterized by a low level of $\mathrm{Fe}(\mathrm{III})$, were the most resistant to bleomycin, a model chemotherapeutic agent whose anticancer activity is associated with iron (20). In contrast, the MCF-7/CDDP resistant cancer cells that have an increased level of intracellular Fe(III) showed the same sensitivity to bleomycin treatment as MCF-7 cells.

MiR-133a targets FTL and increases sensitivity of breast cancer cells to chemotherapeutic agents. Recent reports demonstrating the critical role of iron-regulatory proteins in breast cancer progression (4-7) suggest that targeting these
Table II. Drug sensitivity of parental MCF-7 breast cancer cells and its drug-resistant variants $\mathrm{MCF}-7 / \mathrm{DOX}$ and $\mathrm{MCF}-7 /$ CDDP.

\begin{tabular}{lrcc}
\hline & \multicolumn{3}{c}{ The half maximal inhibitory concentration $\mathrm{IC}_{50}, \mu \mathrm{M}$} \\
\cline { 2 - 4 } Drug & MCF-7 & MCF-7/DOX & MCF-7/CDDP \\
\hline DOX & $4.1 \pm 0.3$ & $23.3 \pm 2.1^{\mathrm{a}}$ & $12.4 \pm 1.2^{\mathrm{a}}$ \\
CDDP & $15.3 \pm 1.3$ & $16.0 \pm 1.0$ & $93.3 \pm 7.0^{\mathrm{a}}$ \\
Bleomycin & $6.4 \pm 0.5$ & $11.3 \pm 0.6^{\mathrm{a}}$ & $4.1 \pm 0.4$ \\
\hline
\end{tabular}

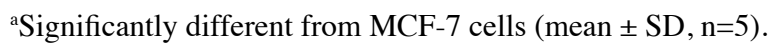

proteins may be a potential therapeutic approach to improve clinical management of breast cancer and overcome resistance of breast cancer cells to chemotherapeutic agents (11). Computational analysis of the 3'-UTR of FTL gene, using the TargetScanHuman, version 6.2, database (www.targetscan. org), revealed the presence of putative binding sites for two microRNAs, miR-22 and miR-133. Recently, Wu et al (21) showed that the level of miR-133a is markedly reduced in breast cancer and is associated with breast cancer progression. The expression of miR-133a was also lower in breast cancer cell lines and displayed $\mathrm{Ct}$ values of $>33$ cycles in MCF-7/ DOX and MCF-7/CDDP cells suggesting that this miRNA is not expressed in drug resistant cells (data not shown). In contrast, the expression of miR-22 was substantially increased in MCF-7 cells resistant to doxorubicin and cisplatin $(22,23)$.

To determine whether or not upregulation of miR-133a affects the FTL levels and increases the sensitivity of drugresistant cells to chemotherapeutic agents, we transfected MCF-7/DOX and MCF-7/CDDP cells with pre-miR-133a. First, we confirmed experimentally that miR-133a targets human FTL mRNA. This was accomplished by transfecting human MDA-MB-231 breast cancer cells, which are characterized by high levels of FTL, with the miR-133a precursor or a siRNA directed against $F T L$. Fig. 4B shows a reduction in the levels of FTL by 76 and $71 \%$, respectively, in the MDA-MB-231 cells transfected with pre-miR-133a precursor and siRNA, as compared to mock-transfected cells. Then, we transfected MCF-7/DOX and MCF-7/CDDP drug-resistant cells with pre-miR-133a and found an ectopic upregulation of miR-133a that resulted in a substantially increased cancer cell sensitivity to doxorubicin and cisplatin. This was evidenced by the fact the $\mathrm{IC}_{50}$ concentration for doxorubicin and cisplatin in MCF-7/DOX and MCF-7/CDDP cells decreased 7.9- and 2.0-fold, respectively (Fig. 4C and D).

\section{Discussion}

Iron is required for normal cell function and is finely regulated by extracellular and intracellular mechanisms responsible for iron homeostasis. In contrast, in cancer cells, the intracellular iron metabolism is profoundly disturbed (5). A number of previous studies have demonstrated a fundamental role of an altered intracellular iron homeostasis in breast cancer. Specifically, breast tumors have aberrant levels of iron- 


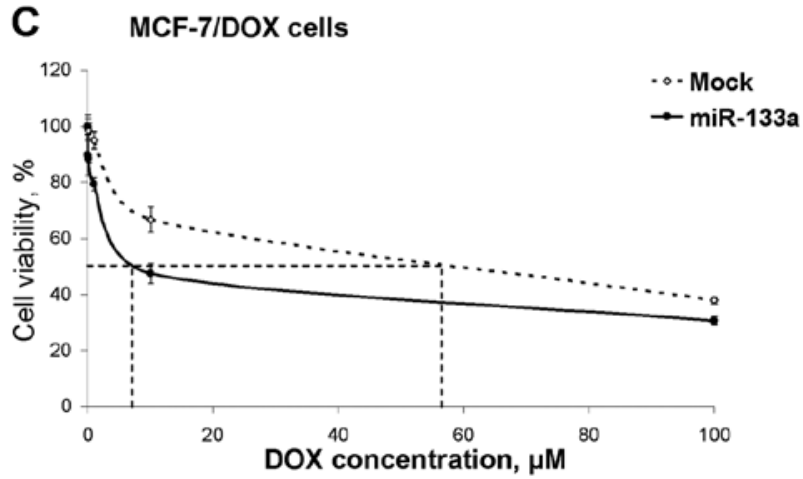

\section{B MDA-MB-231 cells}
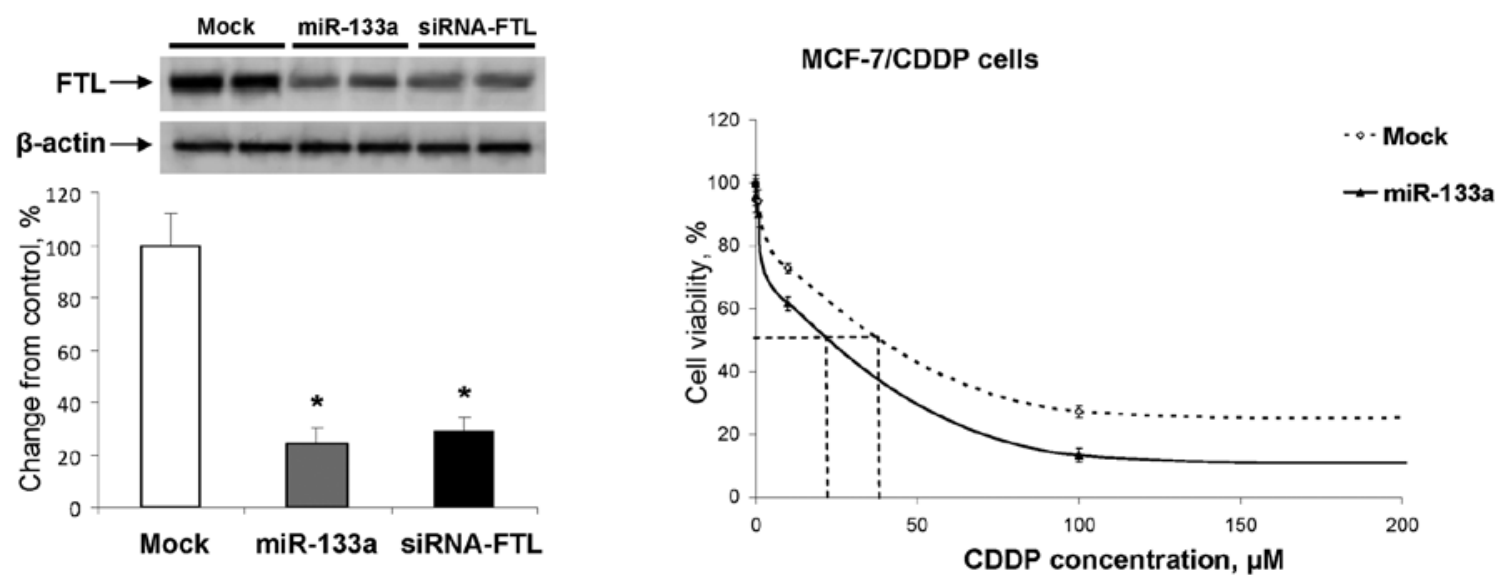

Figure 4. MiR-133a directly targets the human FTL gene. (A) Complementary sequence for miR-133a in the 3'-untranslated region of $F T L$. (B) The level of FTL protein in MDA-MB-231 cells transfected with scrambled RNA oligonucleotide (mock), pre-miR-133a, or siRNA against FTL (siRNA-FTL) for $48 \mathrm{~h}$. The data are presented as percent change in the level of FTL in MDA-MB-231 cells transfected with pre-miR-133a or siRNA-FTL relative to that in mock-transfected MDA-MB-231 cells, which were assigned a value of $100 \%$. Asterisks $(*)$, significantly different from mock-transfected MDA-MB-231 cells $(\mathrm{n}=3)$. (C) Sensitivity of MCF/DOX cells transfected with pre-miR-133a to doxorubicin treatment ( $\mathrm{n}=3$ ). (D) Sensitivity of MCF/CDDP cells transfected with pre-miR-133a to cisplatin treatment $(\mathrm{n}=3)$. For the drug sensitivity analysis, the miR-133a-transfected MCF-7/DOX and MCF-7/CDDP cells were plated at a density of $3 \times 10^{3}$ cells per well in 96 -wells plates and treated with various concentrations of doxorubicin and cisplatin. After $72 \mathrm{~h}$ of incubation, cell survival was analyzed by using the CellTiter-Blue ${ }^{\circledast}$ Cell Viability assay (Promega, Madison, WI, USA). The $\mathrm{IC}_{50}$ values were determined using resulting dose-response curves. The results were reproduced in two independent experiments.

regulatory proteins, including TFR1, ribonucleotide reductase, FTL, FTH1, FPN and hepcidin (4-7,24-27). It is believed that these changes may accelerate tumor growth leading to a more aggressive tumor behavior, metastasis, drug resistance and high recurrence of the disease $(9,17)$.

In this study, we report that human MCF-7 breast cancer cells with an acquired resistance to the chemotherapeutic drugs doxorubicin and cisplatin exhibited substantial alterations in the intracellular iron content and levels of iron-regulatory proteins involved in the cellular uptake, storage and export of iron. The results demonstrate that the levels of intracellular 'free iron' in drug-resistant MCF-7/DOX and MCF-7/CDDP cells were distinctively different. This was evidenced by the fact that the level of intracellular iron in MCF-7/DOX cells was substantially reduced as compared to parental MCF-7 cells, which may be explained by a direct iron-chelating ability of doxorubicin $(28,29)$ and by ability of doxorubicin to interact with iron response elements of FTH1 and FTL mRNAs (30). In contrast, the level of intracellular iron was profoundly increased in MCF-7/CDDP drug-resistant cells.

It is well-established that changes in the cellular iron levels are one of the major causes of the upregulation of iron-regu- latory proteins and ferritins and the translocation of ferritins to the nucleus $(19,31)$. One of the key findings in this study was profoundly increased levels of FTL protein in MCF-7/ DOX and MCF-7/CDDP drug-resistant cells, in general and in the nuclei, in particular. Similarly, in our previous study, increased levels of FTL and FTH1 were found in the aggressive and drug-resistant human MDA-MB-231 breast cancer cells (18). In view of this, we hypothesize that increased levels of FTL may be one of the factors associated with drug-resistance. This suggestion is supported by evidence showing that nuclear ferritins protect DNA from DNA damage-inducing compounds, including DNA-alkylating chemotherapeutic drugs (32).

The increased levels of FTH1 and FTL proteins in breast cancer indicate that these proteins may be used as diagnostic and, more importantly, as prognostic markers for breast cancer $(4,6,26,27)$. Additionally, several reports have shown that FTH1 and FTL may be potential targets in cancer and that their downregulation may substantially increase the efficiency of cancer therapy $(33,34)$. Moreover, it has been suggested that targeted correction of upregulated ferritins may also be a potential approach to overcome resistance of breast cancer 
cells to chemotherapeutic agents (11). The results of the present study, showing that targeted downregulation of FTL protein by miR-133a increases sensitivity of MCF-7/DOX and MCF-7/CDDP cells to doxorubicin and cisplatin support this suggestion. Additionally, these findings are in good agreement with a recent report by Liu et al (34) demonstrating that silencing of FTH1 by siRNA substantially sensitized tumors to chemotherapy.

In conclusion, the data presented herein point to dysregulated iron metabolism as one of the main factors associated with drug-resistant phenotype of breast cancer cells and indicate that correction of these alterations may substantially improve the efficiency of breast cancer treatment.

\section{Acknowledgements}

The views expressed in this paper do not necessarily represent those of the U.S. Food and Drug Administration.

\section{References}

1. Siegel R, Naishadham D and Jemal A: Cancer statistics, 2013. CA Cancer J Clin 63: 11-30, 2011.

2. Jemal A, Bray F, Center MM, Ferlay J, Ward E and Forman D: Global cancer statistics. CA Cancer J Clin 61: 69-90, 2011.

3. Gonzalez-Angulo AM, Morales-Vasquez F and Hortobagyi GN: Overview of resistance to systemic therapy in patients with breast cancer. Adv Exp Med Biol 608: 1-22, 2007.

4. Pinnix ZK, Miller LD, Wang W, D'Agostino R Jr, Kute T, Willingham MC, Hatcher H, Tesfay L, Sui G, Di X, Torti SV and Torti FM: Ferroportin and iron regulation in breast cancer progression and prognosis. Sci Transl Med 2: 43ra56, 2010.

5. Torti SV and Torti FM: Ironing out cancer. Cancer Res 71: 1511-1514, 2011.

6. Miller LD, Cofman LG, Chou JW, Black MA, Bergh J, D'Agostino R Jr, Torti SV and Torti FM: An iron gene signature predicts outcome in breast cancer. Cancer Res 71: 6728-6737, 2011.

7. Kwok JC and Richardson DR: The iron metabolism of neoplastic cells: alterations that facilitate proliferation? Crit Rev Oncol Hematol 42: 65-78, 2002.

8. Yu Y, GutierrezE, Kovacevic Z, Saletta F, Obeidy P, Rahmanto YS and Richardson DR: Iron chelators for the treatment of cancer. Curr Med Chem 19: 2689-2702, 2012.

9. Hoke EM, Maylock CA and Shacter E: Desferal inhibits tumor growth and does not interfere with tumoricidal activity of doxorubicin. Free Radic Biol Med 39: 403-411, 2005.

10. Rao VA, Klein SR, Agama KK, Toyoda E, Adaci N, Pommier Y and Shacter EB: The iron chelator Dp44mT causes DNA damage and selective inhibition of topoisomerase II alpha in breast cancer cells. Cancer Res 69: 948-957, 2009.

11. Whitnall M, Howard J, Ponka P and Richardson DR: A class of iron chelators with a wide spectrum of potent antitumor activity that overcomes resistance to chemotherapeutics. Proc Natl Acad Sci USA 103: 14901-14906, 2006.

12. Chekhun VF, Lukyanova NYu, Kovalchuk O, Tryndyak VP and Pogribny IP: Epigenetic profiling of multidrug-resistant human MCF-7 breast adenocarcinoma cells reveals novel hyper- and hypomethylated targets. Mol Cancer Ther 6: 1089$1098,2007$.

13. Kars MD, Iseri OD, Gündüz U, Ural AU, Arpaci F and Molnár J: Development of rational in vitro models for drug resistance in breast cancer and modulation of MDR by selected compounds. Anticancer Res 26: 4559-4568, 2006.

14. Pate KT, Randel NA, Fraser B, Clement MH and Srinivasan C: Measuring 'free' iron levels in Caenorhabditis elegans using low-temperature Fe(III) electron paramagnetic resonance spectroscopy. Anal Biochem 358: 199-207, 2006.
15. McClelland RA, Wilson D and Leake R: A multicentre study into the reliability of steroid receptor immunocytochemical assay quantification. Eur J Cancer 27: 711-715, 1991.

16. Skehan P, Storeng R, Scudiero D, Monks A, McMahon J, Vistica D, Warren JT, Bokesch H, Kenney S and Boyd MR: New colorimetric cytotoxicity assay for anticancer-drug screening. J Natl Cancer Inst 82: 1107-1112, 1990.

17. Ni J and Hollander D: Application of the MTT-assay to functional studies of mouse intestinal intraepithelial lymphocytes. J Clin Lab Anal 10: 42-52, 1996.

18. Shpyleva SI, Tryndyak VP, Kovalchuk O, Starlard-Davenport A, Chekhun VF, Beland FA and Pogribny IP: Role of ferritin alterations in human breast cancer cells. Breast Cancer Res Treat 126: 63-71, 2011.

19. Arosio P, Ingrassia and Cavadini P: Ferritins: a family of molecules for iron storage, antioxidation and more. Biochim Biophys Acta 1790: 589-599, 2009.

20. Dorr RT: Bleomycin pharmacology: mechanism of action and resistance and clinical pharmacokinetics. Semin Oncol 19 (Suppl 5): 3-8, 1992.

21. Wu Z, Wang C, Xiang R, Liu X, Ye S, Yang X, Zhang G, Xu X, Zhu T and Wu Q: Loss of miR133a expression is associated with poor survival of breast cancer and restoration of miR-133a expression inhibited breast cancer growth and invasion. BMC Cancer 12: 51, 2012.

22. Kovalchuk O, Filkowski J, Meservy J, Ilnytskyy Y, Tryndyak VP, Chekhun VF and Pogribny IP: Involvement of microRNA-451 in resistance of the MCF-7 breast cancer cells to chemotherapeutic drug doxorubicin. Mol Cancer Ther 7: 2152-2159, 2008.

23. Pogribny IP, Filkowski JN, Tryndyak VP, Golubov A, Shpyleva SI and Kovalchuk O: Alterations of microRNAs and their targets are associated with acquired resistance of MCF-7 breast cancer cells to cisplatin. Int J Cancer 127: 1785-1794, 2010.

24. Kanojia D, Zhou W, Zhang J, Jie C, Lo PK, Wang Q and Chen H: Proteomic profiling of cancer stem cells from primary tumors of HER2/Neu transgenic mice. Proteomics 12: 3407-3415, 2012.

25. Eswaran J, Cyanam D, Mudravi P, Reddy SD, Pakala SB, Nair SS, Florea L, Fuqua SA, Godbole S and Kumar R: Transcriptomic landscape of breast cancers through mRNA sequencing. Sci Rep 2: 264, 2012.

26. Descotes F, Jézéquel P, Spyratos F, Campion L, Grenot C, Lerebours F, Campone M, Guérin-Charbonnel C, Lanoë D, Adams M andré J, Carlioz A, Martin PM, Chassevent A, Jourdan ML, Guette C, Zanella-Cleon I and Ricolleau G: Identification of potential prognostic biomarkers for nodenegative breast tumours by proteomic analysis: a multicentric 2004 national PHRC study. Int J Oncol 41: 92-104, 2012.

27. Dong X, Yang M, Sun H, Lü J, Zheng Z, Li Z and Zhong L: Combined measurement of CA 15-3 with novel autoantibodies improves diagnostic accuracy for breast cancer. Onco Targets Ther 6: 273-279, 2013.

28. Xu X, Persson HL and Richardson DR: Molecular pharmacology of the interaction of anthracyclines with iron. Mol Pharmacol 68: 261-271, 2005.

29. Xu X, Sutak R and Richardson DR: Iron chelation by clinically relevant anthracyclines: alteration in expression of iron-regulated genes and atypical changes in intracellular iron distribution and trafficking. Mol Pharmacol 73: 833-844, 2008.

30. Canzoneri JC and Oyelere AK: Interaction of anthracyclines with iron responsive elements mRNAs. Nucleic Acids Res 36: 6825-6834, 2008.

31. Thompson KJ, Fried MG, Ye Z, Boyer P and Connor JR: Regulation, mechanisms and proposed function of ferritin translocation to cell nuclei. J Cell Sci 114: 2165-2177, 2002.

32. Alkhateeb AA and Connor JR: Nuclear ferritin: a new role for ferritin in cell biology. Biochim Biophys Acta 1800: 793-797, 2010.

33. Yang DC, Jiang X, Elliot RL and Head JF: Antisense ferritin oligonucleotides inhibit growth and induce apoptosis in human breast carcinoma cells. Anticancer Res 22: 1513-1524, 2002.

34. Liu X, Madhankumar AB, Slagle-Webb B, Sheehan JM, Surguladze N and Connor JR: Heavy chain ferritin siRNA delivered by cationic liposomes increases sensitivity of cancer cells to chemotherapeutic agents. Cancer Res 71: 2240-2249, 2011. 